\title{
Effect of Nitrogen-Doped Carbon Dots (NCDs) on the Characteristics of NCD/MIL-53(Fe) Composite and Its Photocatalytic Performance for Methylene Blue Degradation under Visible Light
}

\author{
Ngoc Tue Nguyen $(\mathbb{D}$, Xuan Truong Nguyen $\mathbb{D}$, Duc-Trung Nguyen, Hong Minh Tran, \\ Thi May Nguyen, and Thuong Quang Tran
}

School of Chemical Engineering, Hanoi University of Science and Technology, Hanoi, Vietnam

Correspondence should be addressed to Thuong Quang Tran; quang.tranthuong@hust.edu.vn

Received 31 May 2021; Accepted 11 September 2021; Published 28 September 2021

Academic Editor: Chin Wei Lai

Copyright (c) 2021 Ngoc Tue Nguyen et al. This is an open access article distributed under the Creative Commons Attribution License, which permits unrestricted use, distribution, and reproduction in any medium, provided the original work is properly cited.

\begin{abstract}
Metal-organic framework composites, which are combined from metal-organic framework and advanced carbon material, have drawn great attention in many fields of application such as environmental remediation and catalysts. Within this paper, the carbon/MIL-53(Fe) composite was fabricated via an in situ synthesis, in which N-containing carbon dots (NCDs) were mixed with MOF precursors' solutions under various ratios before going through the solvothermal stage. It was showed that the introduction of a certain amount of NCDs would affect characteristic features and improve the photocatalytic performance of final products. The optimal doping content of NCDs in NCD/MIL-53(Fe) composite was determined. SEM images showed that the M-140 appeared as hexagonal bipyramid-shaped crystals with an average size of $700 \mathrm{~nm}$. Compared with pristine MIL-53(Fe), the M-140 was more visibly light-responsive, and its calculated band gap energy was approximately $2.3 \mathrm{eV}$. In addition, M-140 catalyst also displayed more excellent photocatalytic activity for Methylene Blue degradation in a $\mathrm{pH}$ range from 5 to 7 . Under optimal conditions, MB was achieved within 60 minutes and the removal rate was nearly $100 \%$ after 5 cycles. The photocatalytic mechanism of the obtained NCD/MIL-53(Fe) composite was discussed.
\end{abstract}

\section{Introduction}

Metal-organic frameworks (MOFs), a class of highly ordered materials consisting of metal ions/clusters and multifunctional organic bridges, have emerged considerable attention in various fields of applications due to their unique properties such as high surface area, structural diversity, and tailorability. In photocatalysis, metal clusters can absorb incident photons to excite electrons, while the organic bridges can function like antennas to harvest incoming light [1]. Fecontaining MOFs, which have Fe oxo-clusters as the metal core, become a promising photocatalyst because $\mathrm{Fe}-\mathrm{O}$ clusters are visible light-responsive. MIL-53(Fe), the typical Fecontaining $\mathrm{MOF}$, is constructed from $\mathrm{FeO}_{6}$ octahedral chain cross-linked 1,4-benzene dicarboxylate (BDC) ligands. The
MIL-53(Fe) has been studied for various applications such as adsorption [2], sensors [3] and photocatalytic degradation contaminants [4], and drug delivery [5] due to its flexible structure making it easier to open/close its pores for adsorption/desorption guest molecules [2].

The number of publications on the MOF composite has increased considerably in recent years. MOF composites, which consist of MOFs and other materials, are easier to synthesize, and they show more advantages and have fewer drawbacks in comparison with the individual components [6]. Some notable MOF composites including MOF/silica, $\mathrm{MOF} /$ metal nanoparticles, MOF/organic polymers, and $\mathrm{MOF} /$ carbon (Table 1) composites have been successfully synthesized. Especially, metal-organic framework- (MOF-) derived carbon materials have attracted great interest in many 


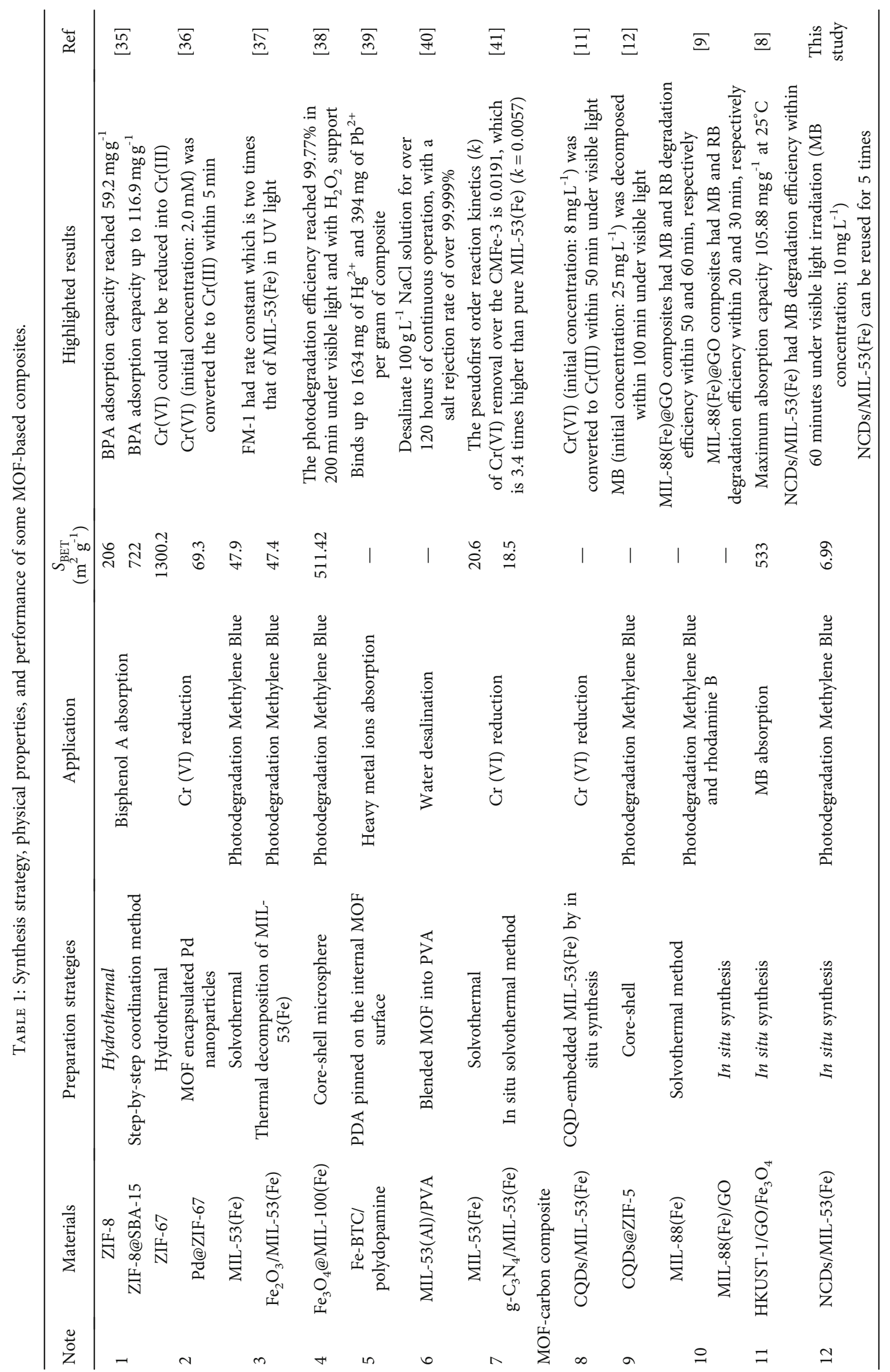


fields of application owing to their nanoporous structure yielding a high surface area and tunable chemical and physical properties [7]. Furthermore, various advanced carbon materials, including CNTs, graphene, C60, carbon quantum dots, carbon fibers, and activated carbon, have been coupled with $\mathrm{MOF}$ to prepare the $\mathrm{MOF} /$ carbon hybrid materials [8-12].

Preparation methods have a great influence on the configuration of the final sample. For example, Lin et al. successfully synthesized MIL-53(Fe)/CQD photocatalyst by solvothermal a mixture comprising MOF precursors and glucose as a carbon source. The results indicated that CQDs were in situ generated inside the pores of MIL-53(Fe) particles, and CQD-embedded MIL-53(Fe) showed a better activity toward $\mathrm{Cr}(\mathrm{VI})$ reduction than MIL-53(Fe) [11]. Another research conducted by $\mathrm{Si}$ et al. showed that the $3-5 \mathrm{~nm}$ in size CQDs could wrap on the surface of ZIF-8 nanoparticles to form a well-designed core-shell structure. Furthermore, the CQDs@ZIF-8 composite exhibited superior photocatalytic performance, while the MB removal rate was 6 times higher than that of ZIF-8 [12]. Meanwhile, Petit et al. had tried to improve the porosity of some typical MOF materials, such as MOF-5, HKUST-1, and MIL-100(Fe), by preparing MOF-graphene oxide (GO) composite via an in situ method. To be more specific, graphene oxide was added to the MOF precursor mixture, and the mixture then underwent the same heating process as the synthesis of pure MOF [13-15]. The authors also mentioned three advantages of the in situ method: (i) functional groups of GO can function as nucleation sites during the formation of MOF, (ii) chemical reactions can lead to synergistic effects between GO and MOF, and (iii) new porosities could be formed because of the irregular arrangement of graphene sheets and MOF [16].

Carbon dots (C-dots or CDs), a member of the nanomaterial class, were first discovered in 2004 by $\mathrm{Xu}$ et al. when the authors purified single-wall carbon nanotubes (SWCNTs) [17]. CDs are also called carbon nanodots, fluorescent carbon NPs, or fluorescent carbon dots $[18,19]$ owing to their interesting physical and optical properties such as nanosized particles, broad optical absorption, multiple photoluminescence spectra, upconverted luminescence, and fast electron transfer capability [20]. These impressive features of C-dots are caused by their structure, which consists of $\mathrm{sp}^{2}$ and $\mathrm{sp}^{3}$ hybrid carbon networks with various functional groups [21]. They have loads of applications such as biometric measurement [18], photocatalyst [22], sensing [19], LED [23], and energy storage devices [24]. Heteroatom-doped carbon dots, for example, N-doped carbon dots, have been widely studied because they show better optical properties in comparison with the original C-dots [22]. Precursors for the synthesis of N-doped CDs are commonly rich-carbon substances (sugar, biomass, and organic compounds) and amine derivatives $\left(\mathrm{NH}_{3}\right.$, Urea, ethanolamine, and 1,2-ethylenediamine) [25]. There are different routes to synthesize carbon dots, such as laser ablation [26], microwave irradiation [21, 27], and hydrothermal synthesis [28], among which the most facile method is microwave irradiation due to lower associated costs and one-step procedure [21]. Recently, C-dots are widely studied in the strategy of designing catalytic systems, in which they function as elec- tron mediators or photosensitizers to enhance the performance of the final catalyst [29].

Herein, in situ synthesis was employed to prepare MIL53(Fe)/carbon composite reported by Petit et al. with some adjustment. Instead of using GO, N-containing carbon dots (NCDs) were employed as a carbon-based component and were prepared from a mixture of citric acid and urea. The effects of the adding amount of NCDs on characteristic features and photocatalytic performance of all samples were investigated. Methylene Blue was chosen as a contaminant for the photocatalytic reaction because it is a widely used textile industry. When the MB concentration in the effluent is too high, it leads to serious water pollution problems such as reducing light transparency, which in turn negatively affects the growth of natural organisms in the aqueous environment. The reaction parameters affecting $\mathrm{MB}$ degradation by the as-prepared catalysts were also determined, including the $\mathrm{pH}$ solution, catalyst, and $\mathrm{H}_{2} \mathrm{O}_{2}$ external dosage.

\section{Experimental Method}

2.1. Materials and Substances. All chemicals and reagents were obtained from commercial suppliers and used without further purification. Ferric chloride hexahydrate ( $>99 \%$, $\mathrm{FeCl}_{3} \cdot 6 \mathrm{H}_{2} \mathrm{O}$ ), terephthalic acid (1,4-BDC, $\left.>98 \%\right)$, Methylene Blue ( $\mathrm{MB},>99 \%)$, hydroperoxide solution $\left(\mathrm{H}_{2} \mathrm{O}_{2}, 30 \%, v / v\right)$, citric acid (CA $>99 \%)$, urea (>99\%), ethyl acetate $(>95 \%)$, hydrochloric acid ( $\mathrm{HCl}, 36-38 \%)$, sodium hydroxide $(\mathrm{NaOH},>96 \%)$, isopropyl alcohol (IPA, >99\%), and ethylenediaminetetraacetic acid disodium dihydrate (EDTA$2 \mathrm{Na},>99 \%, \mathrm{C}_{10} \mathrm{H}_{14} \mathrm{~N}_{2} \mathrm{Na}_{2} \mathrm{O}_{8}$ ) were purchased from Xilong Scientific Company; dimethylformamide (DMF, >99\%) was supplied from Samchun Pure Chemical Co., Ltd.; and pbenzoquinone (BQ, $>99 \%$ ) was obtained from Daejung Chemicals \& Metals Co., Ltd. Distilled water was used for all test experiments.

2.2. Preparation of N-Doped Carbon. The N-doped carbon dots (NCDs) were prepared from Citric acid and urea as precursors via a microwave-assisted method reported by $\mathrm{Qu}$ et al. with some modifications [21]. The molar ratio of citric acid/urea in the precursor mixtures was 1:5 owing to the optical and photoluminescence properties of the obtained NCDs, according to Zholobak et al.'s study [28]. Firstly, citric acid ( $4 \mathrm{~g})$ and urea $(6.3 \mathrm{~g})$ were completely dissolved in distilled water $(20 \mathrm{~mL})$ to form a transparent solution. After that, the solution was heated in a commercial microwave oven at a level of $800 \mathrm{~W}$ for 5 mins, during which the solution changed from a color-less liquid to a dark clustered solid. The solid was then washed with ethyl acetate to remove the residual small molecules and then suspended in $50 \mathrm{~mL}$ distilled water. To remove large or agglomerated particles, the aqueous solution of the NCDs was centrifuged $(4000 \mathrm{rpm}, 20 \mathrm{~min}$ ) and then dried in the vacuum at $60^{\circ} \mathrm{C}$ for 2 days. The resulting dark-brown solid was used for the preparation of NCDs/MIL-53(Fe) in the following stage. 
2.3. Preparation of NC-MIL-53(Fe) Catalysts and Original MIL-53(Fe) Catalyst. MIL-53(Fe) photocatalyst was synthesized via the solvothermal method employed in our previous study with some modifications [30]. MIL-53(Fe) was synthesized from a mixture of $0.83 \mathrm{~g}(5 \mathrm{mmol} \mathrm{TA})$ and $1.35 \mathrm{~g}$ ( $5 \mathrm{mmol}$ ) $\mathrm{FeCl}_{3} \cdot 6 \mathrm{H}_{2} \mathrm{O}$ in $25 \mathrm{~mL}$ DMF. The mixture was transferred into a Teflon-lined stainless-steel autoclave and heated at the temperature of $150^{\circ} \mathrm{C}$ for 5 hours. The lightbrown solid was collected by filtration and subsequently suspended in $\mathrm{H}_{2} \mathrm{O}_{2} 0.1 \mathrm{M}$ solution in $6 \mathrm{~h}$ under visible light irradiation. Afterward, the solution was filtered, and the solid samples were dried in the vacuum at $70^{\circ} \mathrm{C}$ for $2 \mathrm{~h}$ and $150^{\circ} \mathrm{C}$ for $3 \mathrm{~h}$, eventually denoted as M0.

Carbon-based material and MIL-53(Fe) composite or NCDs/MIL-53(Fe) was prepared via in situ synthesis, with a certain amount of NCD solid (e.g., 100, 120, 140, 160, and $180 \mathrm{mg}$ ) added to MIL-53(Fe) precursor solution. Each mixture was sonicated in a water bath for $1 \mathrm{~h}$ before heating to completely suspend NCDs with other components. The final products were named M-100, M-120, M-140, M-160, and $\mathrm{M}-180$, respectively.

2.4. Characterization. XRD patterns were obtained by a Bruker D8 Discovery X-ray Diffractometer using Ni-filtered Cu$\mathrm{K} \alpha$ radiation $(\lambda=0.154056 \mathrm{~nm})$ as the $\mathrm{X}$-ray source. The recorded range of $2 \theta$ was from $5^{\circ}$ to $45^{\circ}$, and the scanning rate was set at $4^{\circ}$ per min. The FTIR spectra were measured by a JASCO FT/IR6300 Spectrometric Analyzer in the range from 400 to $4000 \mathrm{~cm}^{-1}$ at room temperature. UV-vis diffuse reflectance spectra (UV-vis DRS) were recorded by a JACO V-670 UV-vis spectrophotometer with $\mathrm{BaSO}_{4}$ as a reflectance standard. The morphology of the samples was observed with a JEOL JMS-7600F scanning electron microscope (SEM). Elemental mapping of the sample was characterized by energy-dispersive X-ray spectroscopy (EDS, Oxford instruments X-Max). The Brunauer-Emmett-Teller (BET) surface areas and pore size distribution of the samples were determined on a TriStar $3000 \mathrm{~V} 6.07 \mathrm{~A}$ at $77 \mathrm{~K}$ using nitrogen adsorption isotherm.

2.5. Catalytic Degradation Experiments. Textile dye degradation experiments were conducted under visible light. The visible light was generated by an OSRAM Mercury vapor lamp $250 \mathrm{~W}$ through a UV-cutoff filter. $10 \mathrm{mg}$ of sample was suspended into $50 \mathrm{~mL}$ of $10 \mathrm{mg} \mathrm{L}^{-1} \mathrm{MB}$ aqueous solution under a stirring process by magnetic stir at ambient conditions. The reaction mixture was then kept in the dark for 30 minutes to establish the adsorption/desorption equilibrium. After 30 minutes, the mixture was taken out to the visible light and subsequently added a known concentration of $\mathrm{H}_{2} \mathrm{O}_{2}$. The remaining dye concentration in centrifugal liquids was determined by the Hatch UV-vis spectrophotometer model DR 3900 with a $1 \mathrm{~cm}$ quartz cell for every 10 minutes.

The parameters of reactions including the initial $\mathrm{pH}$, concentration of dye solution, and $\mathrm{H}_{2} \mathrm{O}_{2}$ dosage were also studied. To determine the stability of the synthesized NCDs/MIL-53(Fe), the catalyst was recovered from the resulting $\mathrm{MB}$ aqueous by centrifugation and reused for subsequent reactions.

\section{Results and Discussion}

\subsection{Characterization of the Prepared NC/MIL-53(Fe) Samples}

3.1.1. X-Ray Diffraction (XRD). To study the crystalline structure of NCDs, MIL-53(Fe), and NCD/MIL-53(Fe) samples, their XRD patterns were recorded and are plotted in Figure 1(a). Looking at the XRD pattern of NCDs, there were various peaks in the range from $20^{\circ}$ to $27^{\circ}$. These peaks were attributed to the diffraction plane of (002) with highly disordered carbon atoms, and the pattern is in good agreement with some previous reports for C-dots [21]. The XRD pattern of M0 exhibited several characteristic peaks at $9.4^{\circ}, 12.9^{\circ}, 17.4^{\circ}$, and $25.7^{\circ}$ that is consistent with the simulation pattern (Figure 2) as well as the previous literature [4, 30]. Additionally, the well-defined peaks suggested the high crystallinity of MIL-53(Fe) material after immersing in diluted $\mathrm{H}_{2} \mathrm{O}_{2}$ solution $(0.1 \mathrm{M})$ that is as stated in our previous study [30]. The XRD curves of denoted-as M-100, M$120, \mathrm{M}-140, \mathrm{M}-160$, and $\mathrm{M}-180$ samples remained nearly unchanged in terms of MOF's characteristics values, confirming the loading amount of NCDs did not affect the crystallinity of the final products. However, when increasing the amount of NCDs, there was a decrease in the intensity of peak $12.9^{\circ}$, while new peaks at around $17.5^{\circ}$ and $27^{\circ}$ emerged. These noticeable changes indicated that NCDs could change the lattice structure of MIL-53(Fe), corresponding to previous studies $[11,31]$.

3.1.2. Fourier Transform Infrared Spectroscopy (FTIR). To identify functional groups of all obtained catalysts, FTIR spectra of all samples are performed in Figure 1(b). FTIR result of NCDs is in good agreement with previous studies $[21,27]$. In detail, there were two absorption peaks in the range from 3000 to $3250 \mathrm{~cm}^{-1}$ assigned to the asymmetric $\left(\vartheta_{\text {as }}(\mathrm{N}-\mathrm{H})\right)$ and symmetric $\left(\vartheta_{\mathrm{s}}(\mathrm{N}-\mathrm{H})\right)$ vibration of $-\mathrm{NH}_{2}$ groups. The absorption peaks around 1697 and $1574 \mathrm{~cm}^{-1}$ were assigned to $\vartheta(\mathrm{C}=\mathrm{O})$, while the peak at $1398 \mathrm{~cm}^{-1}$ corresponded to $\mathrm{C}-\mathrm{N}$ stretching. Looking at the black curve of the M0 sample, the characteristic absorption peaks that appeared at $1622,1524,1375,748$, and $525 \mathrm{~cm}^{-1}$ matched well with the literature $[4,30,32]$. The $1524 \mathrm{~cm}^{-1}$ peak and the $1375 \mathrm{~cm}^{-1}$ peak were attributed to asymmetric $\left(\vartheta_{\text {as }}(\mathrm{C}-\right.$ $\mathrm{O})$ ) and symmetric $\left(\vartheta_{\mathrm{s}}(\mathrm{C}-\mathrm{O})\right)$ vibrations of carboxyl groups, respectively. The $\mathrm{C}-\mathrm{H}$ stretching vibrations of benzene were represented by a sharp peak at $748 \mathrm{~cm}^{-1}$, while the absorption peak at $525 \mathrm{~cm}^{-1}$ corresponds to the $\mathrm{Fe}-\mathrm{O}$ stretching, implying the formation of Fe-oxo bond between the $\mathrm{Fe}^{3+}$ and the carboxylic groups. From FTIR spectra of NCD/MIL-53(Fe) samples, the characteristic peaks of pure MIL-53(Fe) remained unchanged, while there was an increase in the intensity of $\mathrm{C}=\mathrm{O}$ stretching vibration $\left(\sim 1700 \mathrm{~cm}^{-1}\right)$. The increasing intensity suggested that NCDs embedded in NCD/MIL-53(Fe) particles since $\mathrm{C}=\mathrm{O}$ vibration was previously mentioned as $\mathrm{NCD}$ characteristic feature.

3.1.3. Optical Properties of NCDs/MIL-53(Fe). Figure 3 illustrates the UV-vis spectra of the NCD solution, the UV-vis DRS patterns of the photocatalytic materials, and their band gap energy. Firstly, NCD solution exhibited a broad optical 

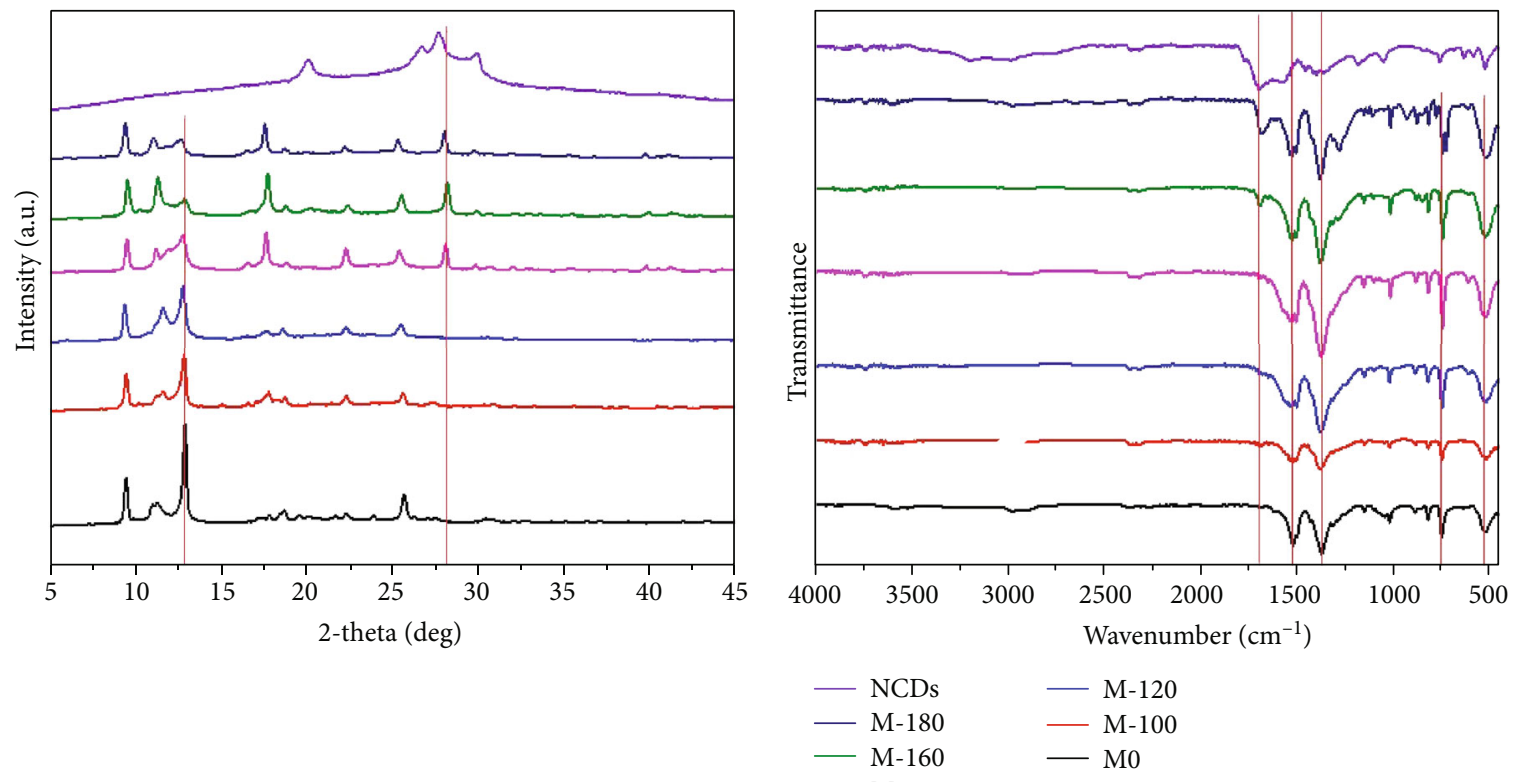

(a)

(b)

FIgure 1: (a) PXRD patterns and (b) FT-IR spectra of MIL-53(Fe) and NCDs/MIL-53(Fe).

absorption in the range of 200 to $500 \mathrm{~nm}$ with three peaks at $270 \mathrm{~nm}, 325 \mathrm{~nm}$, and $410 \mathrm{~nm}$ (Figure $3(\mathrm{a})$ ). The solution also had a yellowish color when being illustrated by daylight. In terms of the UV-vis DRS of M0, the black pattern had the maximum absorption band at around $350 \mathrm{~nm}$ (Figure 3(b)), which could be ascribed to the ligand-tometal charge transfer (LMCT) of $\mathrm{O}(\mathrm{II}) \rightarrow \mathrm{Fe}(\mathrm{III})$ [4, 30, 32]. The absorption edge was at around $420 \mathrm{~nm}$, and the optical band gap was calculated at approximately $2.7 \mathrm{eV}$ (Figure $3(\mathrm{c})$ ) by the relation $E_{\mathrm{g}}=1240 / \lambda$, corresponding to previous studies $[4,30,32]$. Meanwhile, the absorption edge of NCD/MIL-53(Fe) samples was shifted to the longer wavelength region compared with pure MIL-53(Fe). Furthermore, M-140 had the highest background absorption among five NCD/MIL-53(Fe) catalysts (Figure 3(b)), with the maximum absorption band at around $400 \mathrm{~nm}$. In terms of band gap energy, there was a gradual increase in the calculated value as NCDs were added from none to $140 \mathrm{mg}$. However, a slight drop in band gap energy was observed as the adding amount of NCDs rose to $180 \mathrm{mg}$. The sample having the highest estimated bandgap was M-140 $(\sim 2.3 \mathrm{eV})$, corresponding to the changes in UV-vis DRS results of all NCD/MIL-53(Fe) samples. Another noticeable point was the presence of a small characteristic peak in the range of $300-500 \mathrm{~nm}$ happening in the patterns of $\mathrm{M}-100$ and M-120 but not in the other NCD/MIL-53(Fe) patterns. This peak was assigned to the $\mathrm{d}-\mathrm{d}$ transition $\left({ }^{6} \mathrm{~A}_{1 \mathrm{~g}} \rightarrow 4 \mathrm{~A}_{1 \mathrm{~g}}\right.$ $+4 \mathrm{Eg}(\mathrm{G}))$ of $\mathrm{Fe}^{3+}$ ion [32]. The peak for the d-d transition only happened in the absorption spectra of M-100 and M120. This phenomenon could be explained by the formation of defective Fe clusters. The presence of a small amount of NCDs before the formation of MIL-53(Fe) lattice would cause some defective Fe clusters which could not bond with fully six ligands BDC. As a result, the missing linker Fe clus-

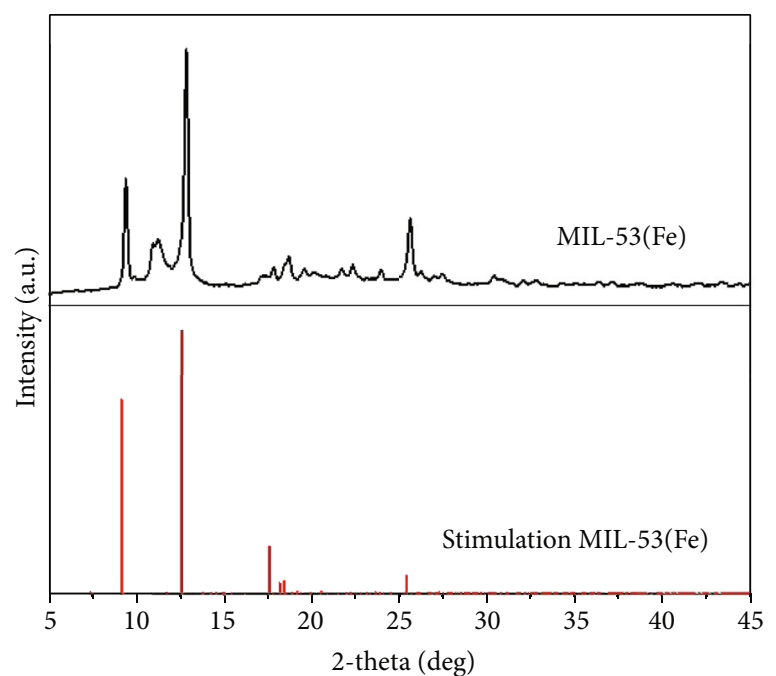

FIgURE 2: XRD patterns of MIL-53(Fe) and the simulated XRD pattern for the MIL-53 structure.

ters would form complexes, leading to the $\mathrm{d}$-d transition presence in UV-vis DRS. When the amount of NCDs was $140 \mathrm{mg}$ and more, Fe clusters would form stable bonds with NCDs and BDC linkers, meaning that characteristics of defective Fe clusters were disappeared.

3.1.4. Scanning Electron Microscopy (SEM) and EnergyDispersive X-Ray Spectroscopy. The morphology and particle size of the MIL-53(Fe) and NCD/MIL-53(Fe) composite samples were examined by scanning electron microscopy (SEM) at different magnifications (Figure 4). As featured in Figure 4(a), pure MIL-53(Fe) was microrods, with an 


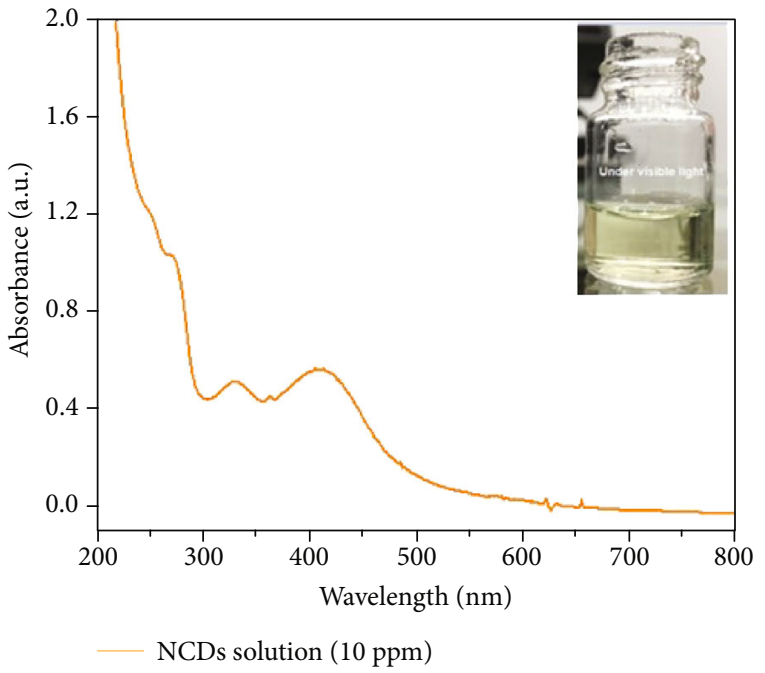

(a)

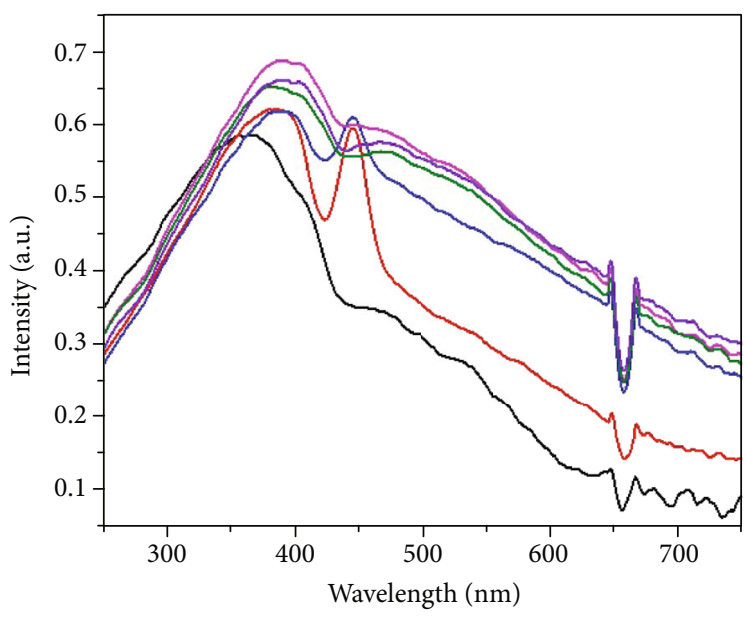

(b)

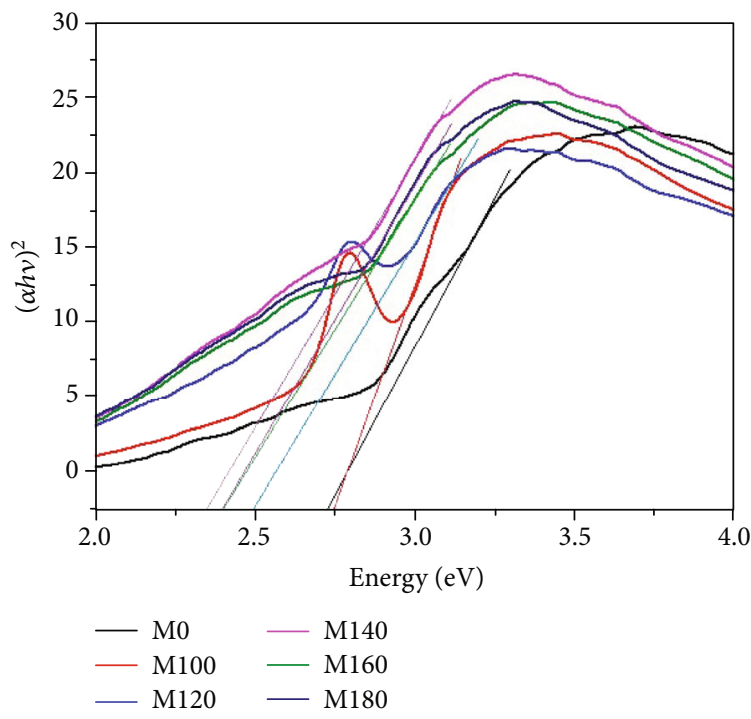

(c)

FIGURE 3: (a) UV-vis absorption spectrum of NCD solution (10 ppm), (b) UV-vis DRS spectra, and (c) calculated band gap of MIL-53(Fe) and NCD/MIL-53(Fe) samples.

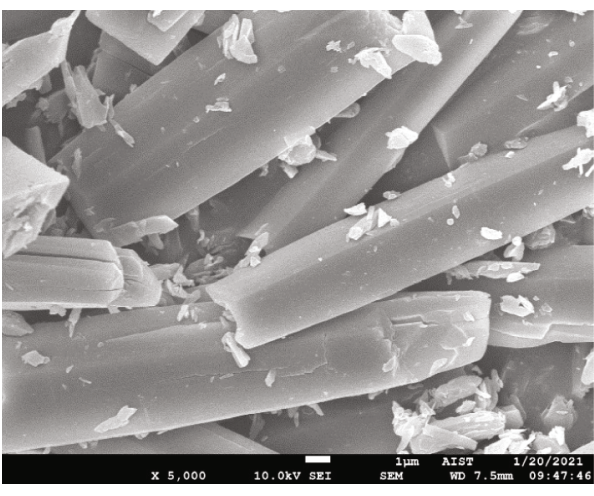

(a)

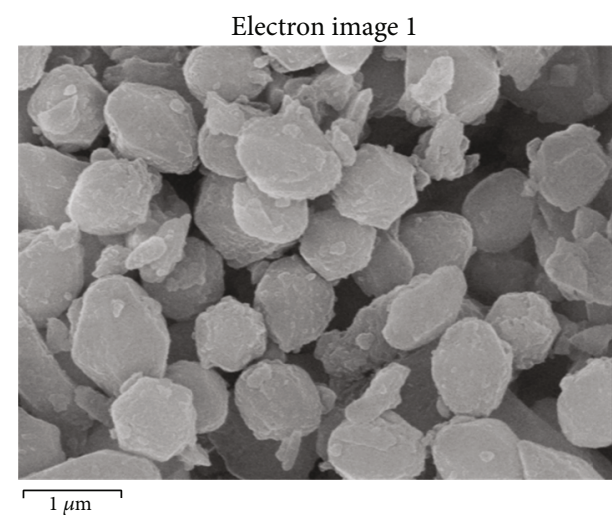

(b)

Figure 4: (a) SEM images of MIL-53(Fe) particles at different magnifications; (b) SEM images of NCD/MIL-53(Fe) composite particles (M-140). 
EDS layered image 1

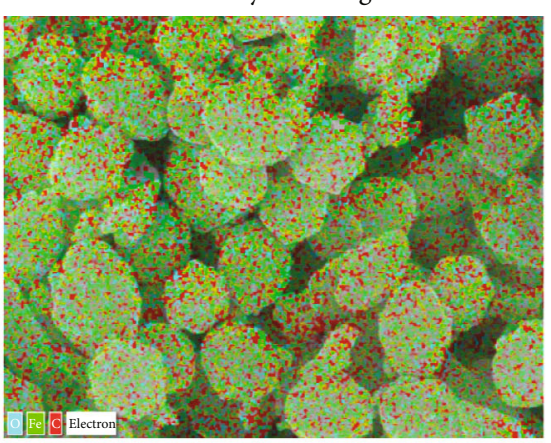

$\mathrm{CK} \alpha 1 \_2$

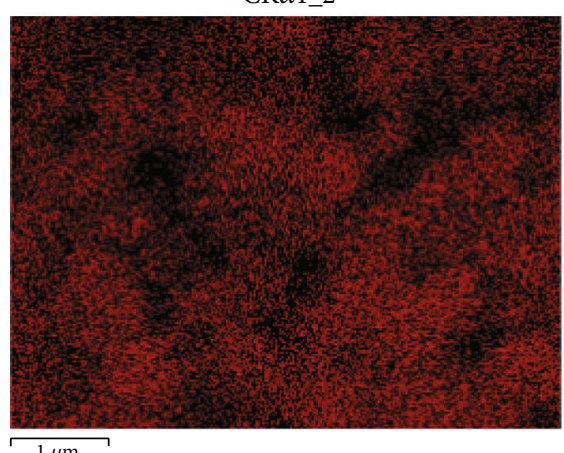

Figure 5: EDS mapping of M-140 samples.
$\mathrm{O} \mathrm{K} \alpha 1$

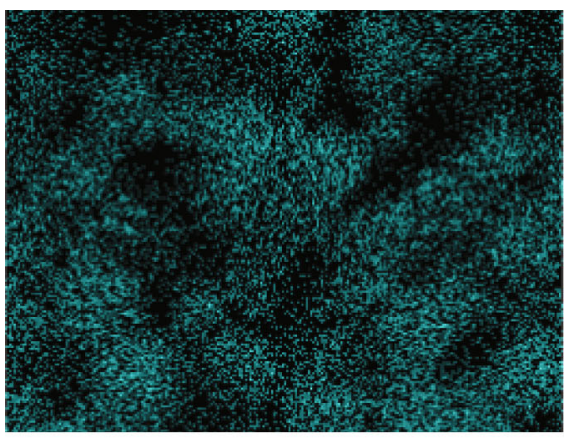

$\mathrm{Fe} \mathrm{K} \alpha 1$

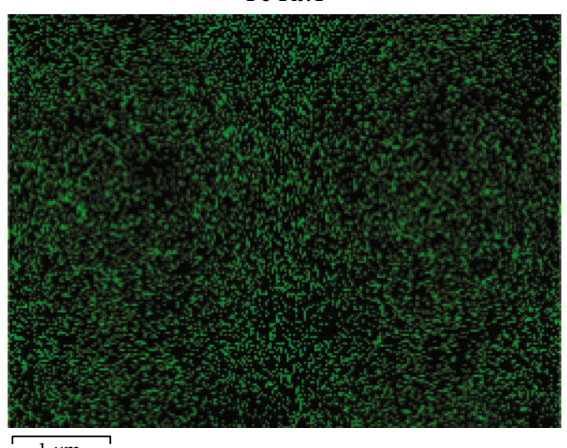

TABLe 2: Specific surface area and porous structure of M0 and M-140.

\begin{tabular}{lcccc}
\hline Samples & Langmuir surface area $\left(\mathrm{m}^{2} \mathrm{~g}^{-1}\right)$ & BET surface area $\left(\mathrm{m}^{2} \mathrm{~g}^{-1}\right)^{\mathrm{a}}$ & Pore volume $\left(\mathrm{cm}^{3} \mathrm{~g}^{-1}\right)^{\mathrm{b}}$ & ${\text { Pore size }(\mathrm{nm})^{\mathrm{c}}}^{\mathrm{a}}$ \\
\hline M0 & 13.35 & 9.77 & 0.03 & 34.13 \\
M140 & 9.75 & 6.63 & 0.001 & 21.09 \\
\hline
\end{tabular}

${ }^{\mathrm{a}} \mathrm{BET}$-specific surface; ${ }^{\mathrm{b}}$ total pore volume measured at $\mathrm{P} / \mathrm{P}_{o}=0.99$; ${ }^{\mathrm{c}}$ pore diameter calculated from the adsorption branch of the isotherm using the $\mathrm{BJH}$ method.

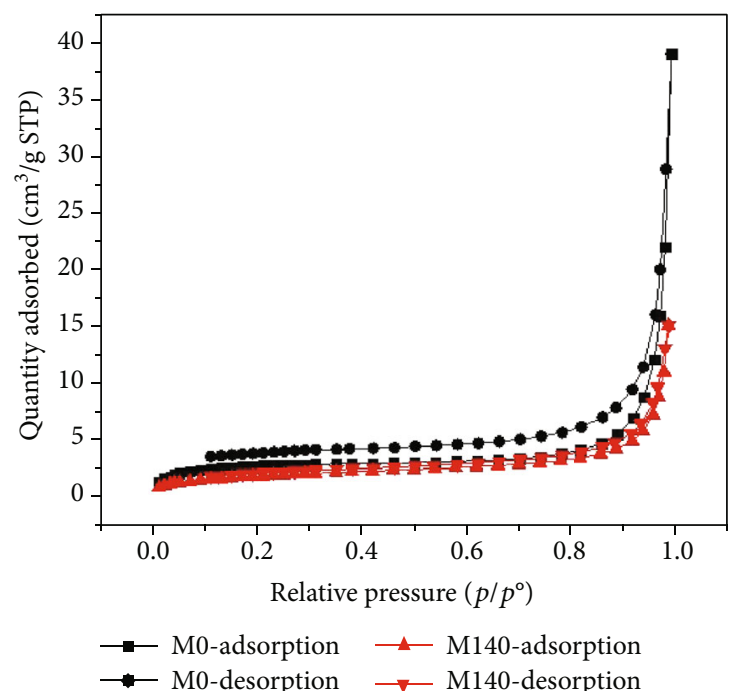

(a)

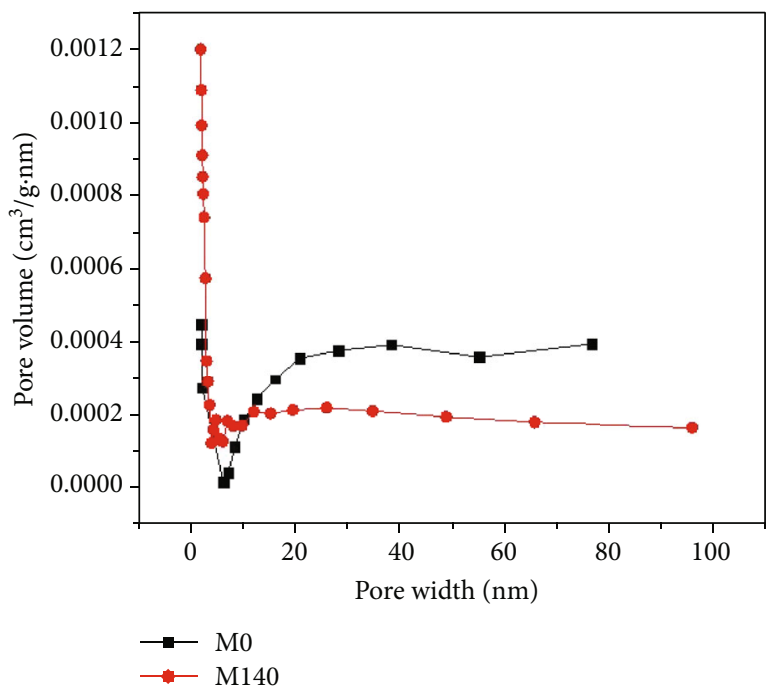

(b)

Figure 6: (a) Nitrogen adsorption-desorption isotherms of M0 and M-140. (b) The Barrett-Joyner-Halenda (BJH) mesoporous size distribution of MIL-53(Fe) and NCDs/MIL-53(Fe). 


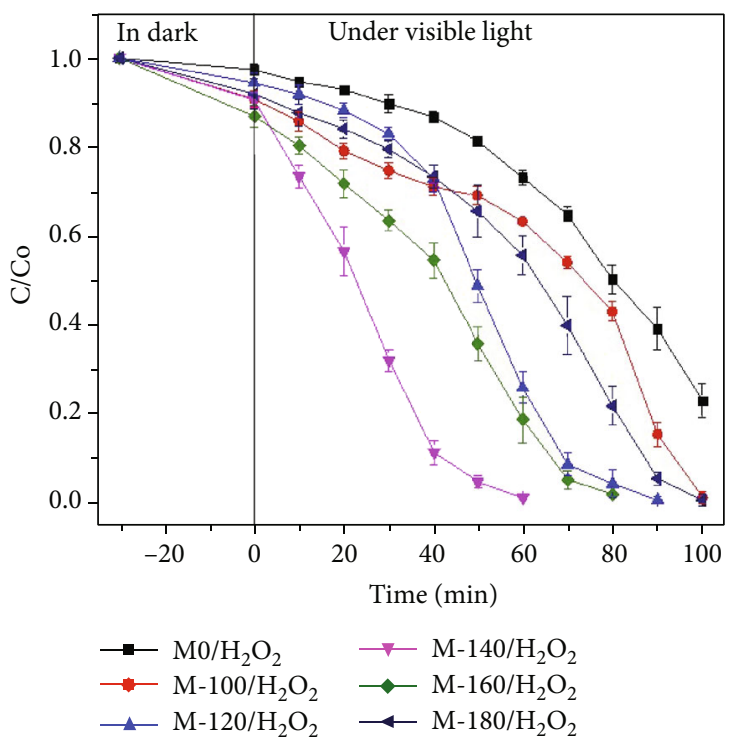

(a)

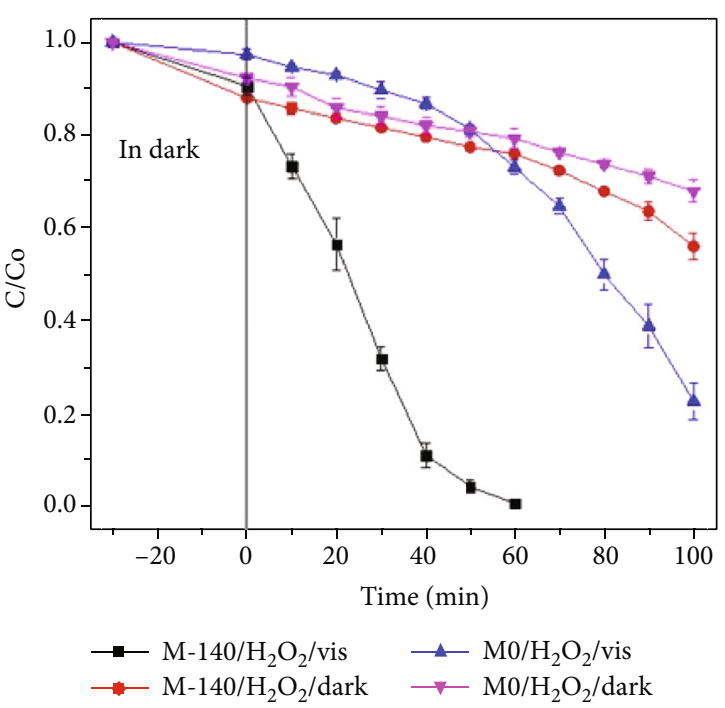

(b)

Figure 7: (a) The degradation of MB in different conditions. (b) The degradation of MB using M0 and M-140 in different conditions (experimental conditions: $\mathrm{MB}, 10 \mathrm{mg} \mathrm{L}^{-1} ; \mathrm{H}_{2} \mathrm{O}_{2}, 5 \mathrm{mM}$; and catalyst, $0.2 \mathrm{gL}^{-1}$ ).

average size of $4 \mu \mathrm{m}$ in width and several tens of micrometers in length, adhering to earlier reports for MIL-53(Fe) [4, 30, 32]. Meanwhile, NCDs/MIL-53(Fe) not only had few microbars but also had a myriad of hexagonal-bipyramid-like particles about $700-1000 \mathrm{~nm}$ in size (Figure 4(b)). The particle size of M-140 was much smaller than that of M0. Furthermore, the surface of M-140 nanoparticles looked very rough unlike the smooth surface of MIL-53(Fe) microrods. This rough surface indicated the presence of $\mathrm{C}$-dots with an average size of around $10 \mathrm{~nm}$. The changes in the morphology and particle size also suggested that the presence of NCDs limited space for the formation of the Fe-BDC bonding network, leading to produce smaller particles.

The EDS elemental mapping was used to measure the distribution of the Fe element, and the results are plotted in Figure 5. It is noticeable that iron elements were uniformly dispersed in the bipyramid-like particles confirming the interaction between MOF and C-dots.

The porous properties of M0 and M-140 were compared to each other by $\mathrm{N}_{2}$ adsorption-desorption isotherms at $77 \mathrm{~K}$, and the full data is illustrated in Table 2 and Figure 6. It can be seen from Table 2 that surface area and porous structure values of M-140 were lower than the data for M0. It happened due to the presence of closely packed NCDs that reduce the porosity of M-140 comparing to pristine MIL-53(Fe).

3.2. The Photocatalytic Activity Analyses. The photocatalytic performance of pristine MIL-53(Fe) and NCD/MIL-53(Fe) samples was studied through $\mathrm{MB}$ degradation experiments, and the results are shown in Figure $7(\mathrm{a})$. In detail, about $80 \%$ of $\mathrm{MB}$ concentration was removed within 100 minutes when the M0 is present in the solution. Meanwhile, MB was completely decomposed in the same amount of time with the presence of NCD/MIL-53(Fe) samples. Therefore,

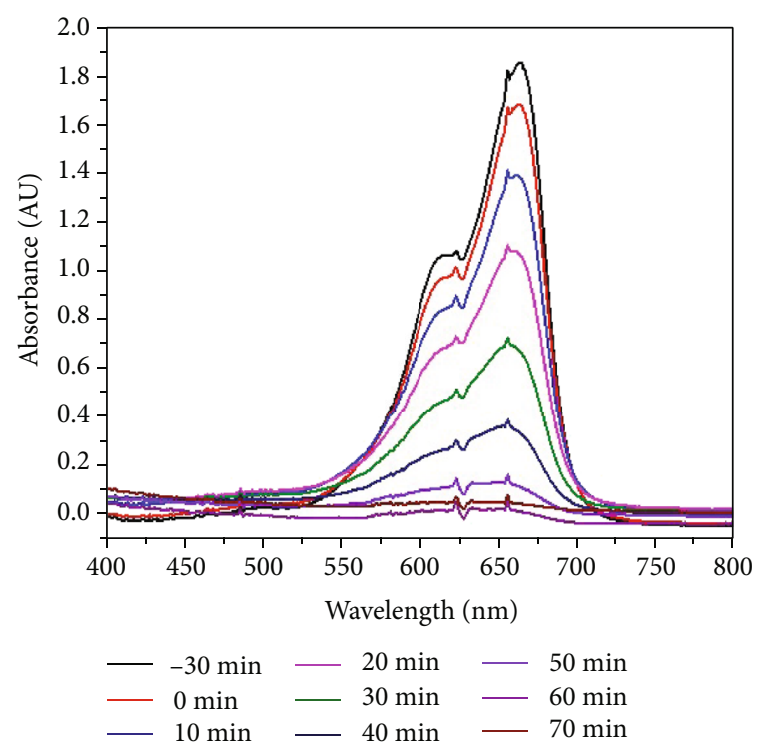

FIgURe 8: The UV-vis spectral changes of $\mathrm{MB}$ during the photodegradation over M-140 (experimental conditions: $\mathrm{MB}$, $10 \mathrm{mg} \mathrm{L}^{-1} ; \mathrm{H}_{2} \mathrm{O}_{2}, 5 \mathrm{mM} ; \mathrm{pH} 7$ and catalyst, $0.2 \mathrm{~g} \mathrm{~L}^{-1}$ ).

NCD/MIL-53(Fe) samples exhibited superior photocatalytic efficiency over the pristine MIL-53(Fe), confirming the synergistic effect between NCDs and MIL-53(Fe). Moreover, the catalytic activity of NCDs/MIL-53(Fe) was affected by adjusting the loading amount of NCDs. It can be seen that the degradation efficiency increased considerably as the loading amount of carbon material increased gradually from $100 \mathrm{mg}$ to $140 \mathrm{mg}$, with the best performance belonged to M-140 sample. The MB removal rate was nearly $100 \%$ within 60 minutes, and the UV-vis absorption spectrum of the reaction at regular intervals of time is plotted in 


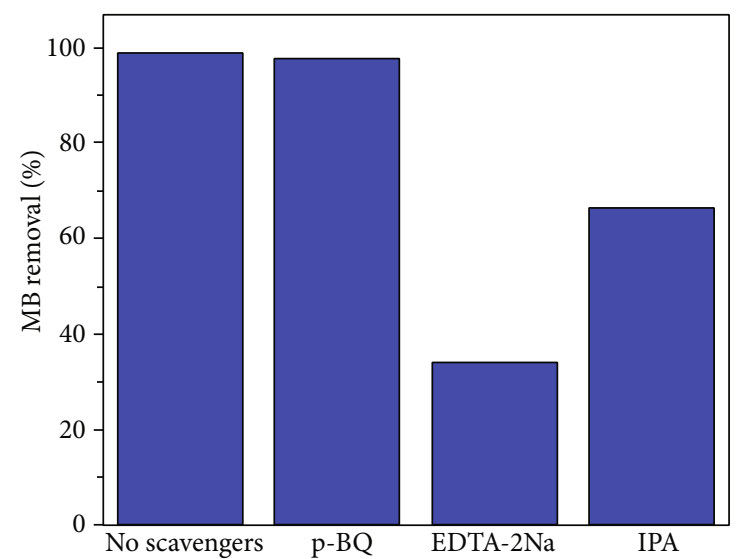

(a)

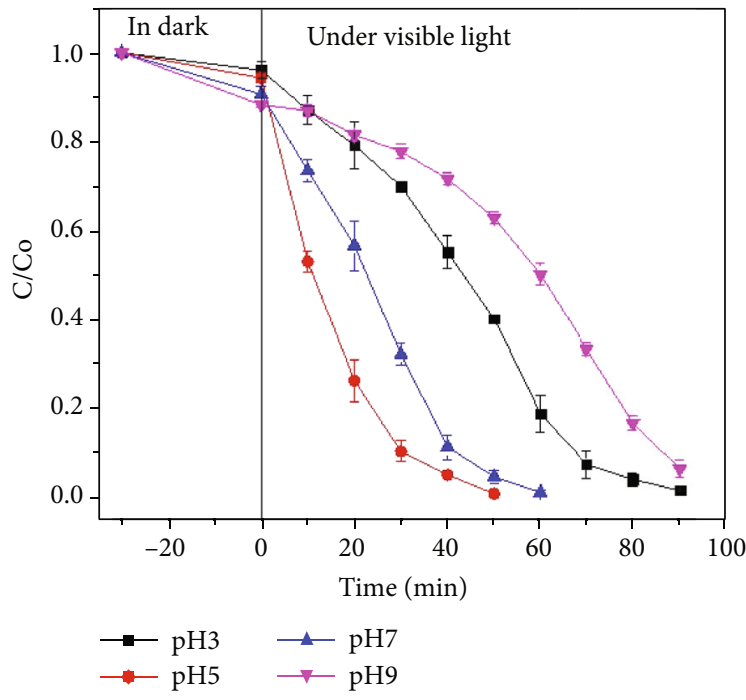

(b)

Figure 9: (a) Effect of different scavengers on the degradation of MB. (b) Effect of initial $\mathrm{pH}$ on the degradation of MB (experimental conditions: $\mathrm{MB}, 10 \mathrm{mg} \mathrm{L}^{-1} ; \mathrm{H}_{2} \mathrm{O}_{2}, 5 \mathrm{mM}$; and $\left.\mathrm{M}-140,0.2 \mathrm{~g} \mathrm{~L}^{-1}\right)$.

Figure 8. However, when the amount of NCDs continuously accelerated from 140 to $180 \mathrm{mg}$, the photocatalytic performance surprisingly decreased. The results were consistent with Lin et al.'s study, even though different precursors were employed in this study. When NCDs acted like an excessive cocatalyst, it could shield incident light photons which were supposed to excite electrons of a metal cluster; as the result, the degradation efficiency reduced; thus, the degradation efficiency reduced [11]. The observed photocatalytic results of all NCD/MIL-53(Fe) samples were also compatible with UV-vis DRS and calculated band gap energy in the previous part of this paper.

Several MB degradation experiments in different conditions were conducted to compare the catalytic ability of M0 and M-140 (Figure 7(b)). As mentioned in the previous part of this paper, the photocatalytic performance of M-140 was better than that of M0. However, almost no change in the catalytic performance of M0 and M-140 was observed under visible light absence conditions. It indicated that the role of NCDs in the NCD/MOF catalyst system would be photosensitizers to enhance the photocatalytic capacity of the final product.

3.3. Mechanism of Degradation by $\mathrm{C}$-dots/MIL-53(Fe) as the Photocatalyst. The catalytic mechanism of NCDs/MIL$53(\mathrm{Fe})$ would be thoroughly studied by investigating the presence of active species generated during degradation $\mathrm{MB}$ reaction (Figure 9(a)). In this part, various scavengers, such as p-benzoquinone (p-BQ), isopropanol (IPA), and EDTA-2Na, were used to capture active species including $\mathrm{O}_{2}{ }^{-},{ }^{\circ} \mathrm{OH}$, and $\mathrm{h}+$. As shown in Figure 9(a), there were significant declines in $\mathrm{MB}$ removal efficiency with the addition of IPA and EDTA-Na, verifying that ${ }^{\circ} \mathrm{OH}$ and $\mathrm{h}+$ were the two prevalent species in the MB degradation mechanism. Meanwhile, BQ did not affect the MB efficiency of M-140 sample, indicating species ${ }^{\circ} \mathrm{O}_{2}{ }^{-}$was inconsiderable.
Based on the results of the scavenger reactions and other results, the mechanism of the NCD/MIL-53(Fe) catalyst system is proposed in Figure 10. Under visible light irradiation, NCDs could capture incident photons with longer wavelengths $(>350 \mathrm{~nm})$ to form electron-hole pairs. In the meantime, MIL-53(Fe) could absorb photons with shorter wavelengths $(<350 \mathrm{~nm})$ to excite electrons. Due to the relative band alignment between NCDs and MIL-53(Fe), the migration of electrons from NCDs to MIL-53(Fe) and the positive charge transfer to NCDs happen, consequently reducing charge recombination. The excited electrons react with external $\mathrm{H}_{2} \mathrm{O}_{2}$ to generate ${ }^{\circ} \mathrm{OH}$ radicals. Both positive holes $(\mathrm{h}+)$ and ${ }^{\circ} \mathrm{OH}$ radicals cause pollutant degradation, confirmed by the results of scavenger reactions. In addition, Felton reaction also is involved in the contaminant decomposition as $\mathrm{Fe}$ (III) on the surface of $\mathrm{Fe}$-containing catalyst could react with $\mathrm{H}_{2} \mathrm{O}_{2}$ to generate ${ }^{\circ} \mathrm{OH}$ radicals following equations (1) and (2) [4]. Overall, the NCD/MIL-53(Fe) composites are more visible light-responsive, have better charge separation, and exhibit more excellent MB photodegradation activity.

$$
\begin{gathered}
\mathrm{Fe}(\mathrm{III})+\mathrm{H}_{2} \mathrm{O}_{2} \longrightarrow \mathrm{Fe}(\mathrm{II})+\mathrm{HOO}^{\bullet}+\mathrm{H}^{+} \\
\mathrm{Fe}(\mathrm{II})+\mathrm{H}_{2} \mathrm{O}_{2} \longrightarrow \mathrm{Fe}(\mathrm{III})+{ }^{\cdot} \mathrm{OH}+\mathrm{OH}^{-}
\end{gathered}
$$

3.3.1. Effect of $\mathrm{pH}$. The influence of $\mathrm{MB}$ solution's initial $\mathrm{pH}$ on catalyst performance was also investigated (Figure 9(b)). The MB degradation efficiency in $\mathrm{pH} 5$ and 7 solutions was nearly the same, and $\mathrm{MB}$ was completely decomposed within $60 \mathrm{~min}$ for both cases. However, there were noticeable decreases in the degradation $\mathrm{MB}$ performance in $\mathrm{pH} 9$ cases, while the remaining $\mathrm{MB}$ concentration was $50 \%$ after 60 minutes under visible light irradiation. The phenomenon in alkaline solution could be due to the deprotonation of the oxygen-containing functional groups on the surface of the NCDs, inducing the increase of surface negative charge. 


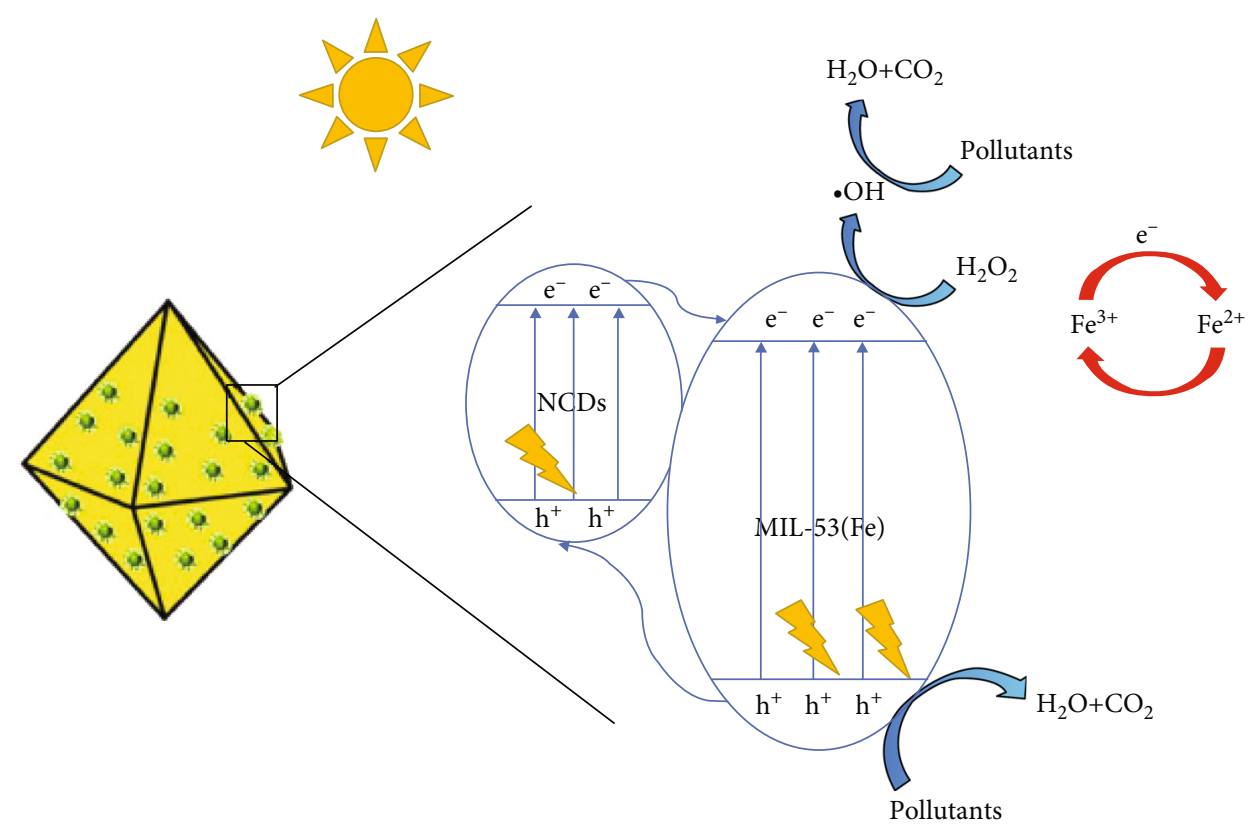

Figure 10: Mechanism scheme of NCDs/MIL-53(Fe) in photocatalytic degradation of pollutants.

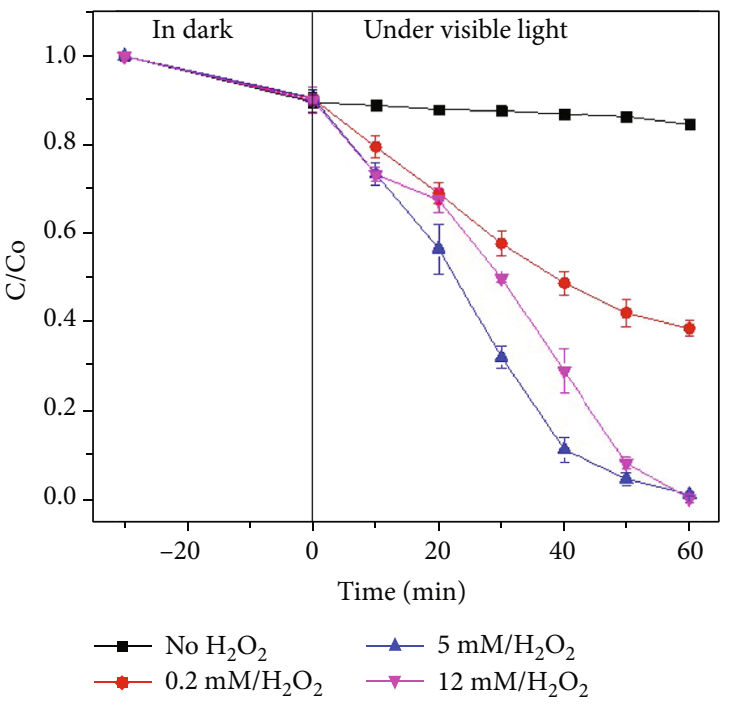

(a)

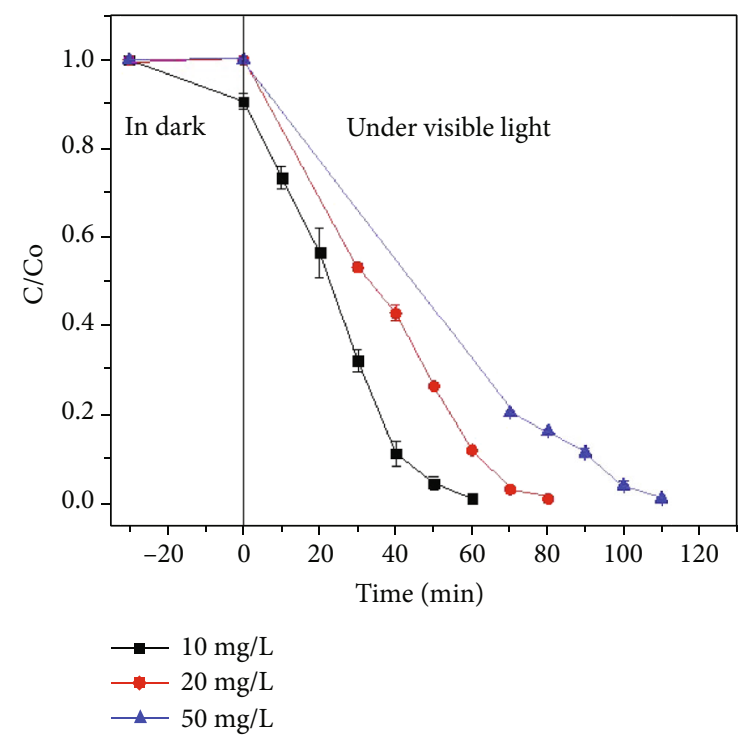

(b)

Figure 11: (a) Effect of $\mathrm{H}_{2} \mathrm{O}_{2}$ concentration (experimental conditions: $\mathrm{MB}, 10 \mathrm{mg} \mathrm{L}{ }^{-1}$; $\mathrm{pH}$, 7; and M-140, $0.2 \mathrm{~g} \mathrm{~L}^{-1}$ ). (b) Effect of MB initial concentration (experimental conditions: $\mathrm{pH}, 7 \mathrm{H}_{2} \mathrm{O}_{2}, 5 \mathrm{mM}$; and $\mathrm{M}-140,0.2 \mathrm{gL}^{-1}$ ).

This caused the shift of the Fermi level, inhibited the electron transition, and resulted in the deceleration in $\mathrm{MB}$ degradation performance [33]. Meanwhile, the MB degradation performance in $\mathrm{pH} 3$ condition slightly decreased, while the MB had removal reached only $80 \%$ after 60 minutes of visible irradiation. For $\mathrm{pH} 3$ case, the decline of decomposing efficiency happened due to the intramolecular and intermolecular hydrogen bond, leading to the strong vibrational coupling of the $-\mathrm{OH}$ functional groups in the surface state of NCDs [34]. Another reason could be the protonation of amine groups that would partially affect the migration of electrons from NCDs to MIL-53(Fe). In general, the M-140 model was capable of decomposing $\mathrm{MB}$ dye in a $\mathrm{pH}$ range of 3 to 9; the most effective was in the range 5-7.

3.3.2. Optimal Degradation Reaction. To optimize the amount of external $\mathrm{H}_{2} \mathrm{O}_{2}$ for $\mathrm{MB}$ decomposition reaction, the $\mathrm{H}_{2} \mathrm{O}_{2}$ concentration was varied from 0 to $12 \mathrm{mM}$ (Figure 11(a)). It was noticeable that the MB degradation rate was only $20 \%$ with the presence of M-140 catalyst and with the absence of $\mathrm{H}_{2} \mathrm{O}_{2}$ within 60 minutes. Meanwhile, about $40 \%$ and nearly $100 \%$ of the dye were decomposed 


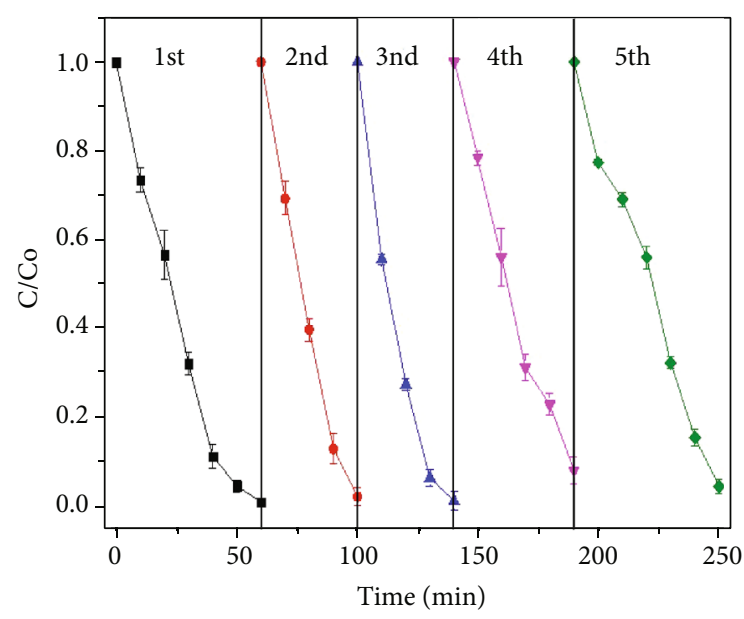

FIGURE 12: Recycling and reuse of M-140 photocatalyst for the removal of Methylene Blue (experimental conditions: $\mathrm{MB}$, $10 \mathrm{mg} \mathrm{L}^{-1} ; \mathrm{pH}, 7 ; \mathrm{H}_{2} \mathrm{O}_{2}, 5 \mathrm{mM}$; and $\mathrm{M}-140,0.2 \mathrm{~g} \mathrm{~L}^{-1}$ ).

with the addition of $0.2 \mathrm{mM} \mathrm{H}_{2} \mathrm{O}_{2}$ and $5 \mathrm{mM} \mathrm{H}_{2} \mathrm{O}_{2}$, respectively. However, with the same added volume, when the $\mathrm{H}_{2} \mathrm{O}_{2}$ concentration increased from $5 \mathrm{mM}$ to $12 \mathrm{mM}$, there was almost no significant change in the degradation efficiency. This has been explained in previous studies that the residual $\mathrm{H}_{2} \mathrm{O}_{2}$ molecule captures hydroxyl ${ }^{\circ} \mathrm{OH}$ radicals to form hydroperoxyl $\left({ }^{\circ} \mathrm{OOH}\right)$ free radicals, which possess less oxidative ability $[4,30]$. This result indicates that the optimal concentration of $\mathrm{H}_{2} \mathrm{O}_{2}$ was $5 \mathrm{mM}$.

The initial MB concentration also has a great influence on the catalytic performance of as-prepared material. The $\mathrm{MB}$ concentration increased gradually from $10 \mathrm{mg} \mathrm{L}^{-1}$ to $50 \mathrm{mg} \mathrm{L}^{-1}$, with $10 \mathrm{mg}$ of $\mathrm{M}-140$ catalyst and the $5 \mathrm{mM}$ $\mathrm{H}_{2} \mathrm{O}_{2}$ for an individual experiment (Figure 11(b)). The results showed that M-140 completely decomposed $\mathrm{MB}$ in the $20 \mathrm{mg} \mathrm{L}^{-1}$ and $50 \mathrm{mg} \mathrm{L}^{-1}$ solution within 80 and 110 minutes, respectively. It is also indicating that the catalyst NCDs/MIL-53(Fe) is not overwhelmed by $50 \mathrm{mg} \mathrm{L}^{-1}$ dye solution.

Finally, the reusability of the catalyst was studied and is presented in Figure 12. M-140 exhibited a good performance after 5 cycles, indicating the recycling ability of NCDs/MIL$53(\mathrm{Fe})$. However, there was a slight drop in the degradation efficiency of the 5th run, which could be explained by the loss of catalyst while recovering by centrifugation.

\section{Conclusion}

In summary, a series of NCD/MIL-53(Fe) composites with different amounts of $\mathrm{N}$-containing carbon dots have been fabricated via an in situ solvothermal method. It was showed that the introduction of a certain amount of NCDs would affect characteristic features and improve the photocatalytic performance of final products. The optimal doping content of NCDs in NCD/MIL-53(Fe) composite was determined to be $140 \mathrm{mg}$. SEM images showed that the M-140 appeared as hexagonal bipyramid-shaped crystals with an average size of $700 \mathrm{~nm}$. Compared with pristine MIL-53(Fe), the M-140 was more visible light-responsive and its calculated band gap energy was approximately $2.3 \mathrm{eV}$. In addition, M-140 catalyst also displayed more excellent photocatalytic activity for Methylene Blue degradation in a $\mathrm{pH}$ range from 5 to 7 . Under optimal conditions, MB was achieved within 60 minutes and the removal rate was nearly $100 \%$ after 5 cycles.

\section{Data Availability}

The data used to support the findings of this study are included within the article.

\section{Conflicts of Interest}

The authors declare that they have no known competing financial interests or personal relationships that could have appeared to influence the work reported in this paper.

\section{Acknowledgments}

The authors are thankful for the financial support under the International Technology Center Pacific (ITC-PAC) program (US-ARMY). This work and paper were made with Government support under Contract No. FA520920P0139 awarded by the International Technology Center Pacific (ITC-PAC).

\section{References}

[1] D. Wang and Z. Li, "Iron-based metal-organic frameworks (MOFs) for visible-light-induced photocatalysis," Research on Chemical Intermediates, vol. 43, no. 9, pp. 5169-5186, 2017.

[2] T. A. Vu, G. H. le, C. D. Dao et al., "Arsenic removal from aqueous solutions by adsorption using novel MIL-53(Fe) as a highly efficient adsorbent," RSC Advances, vol. 5, no. 7, pp. 5261-5268, 2015.

[3] D. Cheng, X. Li, Y. Qiu et al., "A simple modified electrode based on MIL-53(Fe) for the highly sensitive detection of hydrogen peroxide and nitrite," Analytical Methods, vol. 9, no. 13, pp. 2082-2088, 2017.

[4] L. Ai, C. Zhang, L. Li, and J. Jiang, "Iron terephthalate metalorganic framework: Revealing the effective activation of hydrogen peroxide for the degradation of organic dye under visible light irradiation," Applied Catalysis B: Environmental, vol. 148-149, pp. 191-200, 2014.

[5] D. T. C. Nguyen, H. T. N. Le, T. S. Do et al., "Metal-organic framework MIL-53(Fe) as an adsorbent for ibuprofen drug removal from aqueous solutions: response surface modeling and optimization," Journal of Chemistry, vol. 2019, Article ID 5602957, 11 pages, 2019.

[6] X.-W. Liu, T.-J. Sun, J.-L. Hu, and S.-D. Wang, "Composites of metal-organic frameworks and carbon-based materials: preparations, functionalities and applications," Journal of Materials Chemistry A, vol. 4, no. 10, pp. 3584-3616, 2016.

[7] C. Wang, J. Kim, J. Tang et al., "New strategies for novel MOFderived carbon materials based on nanoarchitectures," Chem, vol. 6, no. 1, pp. 19-40, 2020.

[8] L. Li, X. L. Liu, M. Gao et al., "The adsorption on magnetic hybrid $\mathrm{Fe}_{3} \mathrm{O}_{4} /$ HKUST-1/GO of methylene blue from water solution," Journal of Materials Chemistry A, vol. 2, no. 6, pp. 1795-1801, 2014. 
[9] Y. Wu, H. Luo, and H. Wang, "Synthesis of iron(iii)-based metal-organic framework/graphene oxide composites with increased photocatalytic performance for dye degradation," RSC Advances, vol. 4, no. 76, pp. 40435-40438, 2014.

[10] Y. Yang, L. Ge, V. Rudolph, and Z. Zhu, "In situ synthesis of zeolitic imidazolate frameworks/carbon nanotube composites with enhanced $\mathrm{CO}_{2}$ adsorption," Dalton Transactions, vol. 43, no. 19, pp. 7028-7036, 2014.

[11] R. Lin, S. Li, J. Wang et al., "Facile generation of carbon quantum dots in MIL-53(Fe) particles as localized electron acceptors for enhancing their photocatalytic Cr(vi) reduction," Inorganic Chemistry Frontiers, vol. 5, no. 12, pp. 3170-3177, 2018.

[12] Y. Si, X. Li, G. Yang, X. Mie, and L. Ge, "Fabrication of a novel core-shell CQDs@ZIF-8 composite with enhanced photocatalytic activity," Journal of Materials Science, vol. 55, no. 27, pp. 13049-13061, 2020.

[13] C. Petit and T. J. Bandosz, "MOF-graphite oxide composites: combining the uniqueness of graphene layers and metalorganic frameworks," Advanced Materials, vol. 21, no. 46, pp. 4753-4757, 2009.

[14] C. Petit, J. Burress, and T. J. Bandosz, “The synthesis and characterization of copper-based metal-organic framework/graphite oxide composites," Carbon, vol. 49, no. 2, pp. 563-572, 2011.

[15] C. Petit and T. J. Bandosz, "Synthesis, characterization, and ammonia adsorption properties of mesoporous metalorganic framework (MIL(Fe))-graphite oxide composites: exploring the limits of materials fabrication," Advanced Functional Materials, vol. 21, no. 11, pp. 2108-2117, 2011.

[16] C. Petit and T. J. Bandosz, "Engineering the surface of a new class of adsorbents: metal-organic framework/graphite oxide composites," Journal of Colloid and Interface Science, vol. 447, pp. 139-151, 2015.

[17] X. Xu, R. Ray, Y. Gu et al., "Electrophoretic analysis and purification of fluorescent single-walled carbon nanotube fragments," Journal of the American Chemical Society, vol. 126, no. 40, pp. 12736-12737, 2004.

[18] C. Liu, P. Zhang, F. Tian, W. Li, F. Li, and W. Liu, "One-step synthesis of surface passivated carbon nanodots by microwave assisted pyrolysis for enhanced multicolor photoluminescence and bioimaging," Journal of Materials Chemistry, vol. 21, no. 35, pp. 13163-13167, 2011.

[19] Y. Jiao, Y. Gao, Y. Meng et al., "One-step synthesis of label-free ratiometric fluorescence carbon dots for the detection of silver ions and glutathione and cellular imaging applications," ACS Applied Materials \& Interfaces, vol. 11, no. 18, pp. 1682216829, 2019.

[20] B. Gayen, S. Palchoudhury, and J. Chowdhury, "Carbon dots: a mystic star in the world of nanoscience," Journal of Nanomaterials, vol. 2019, Article ID 3451307, 19 pages, 2019.

[21] S. Qu, X. Wang, Q. Lu, X. Liu, and L. Wang, "A biocompatible fluorescent ink based on water-soluble luminescent carbon nanodots," Angewandte Chemie International Edition, vol. 51, no. 49, pp. 12215-12218, 2012.

[22] A. Bhati, S. R. Anand, D. Saini, Gunture, and S. K. Sonkar, "Sunlight-induced photoreduction of $\mathrm{Cr}(\mathrm{VI})$ to $\mathrm{Cr}$ (III) in wastewater by nitrogen-phosphorus-doped carbon dots," $n p j$ Clean Water, vol. 2, no. 1, p. 12, 2019.

[23] L. Jin, L. Zhang, L. Yang et al., "Orange-red, green, and blue fluorescence carbon dots for white light emitting diodes," Jour- nal of Materials Science \& Technology, vol. 50, pp. 184-191, 2020.

[24] J.-S. Wei, T.-B. Song, P. Zhang, X.-Q. Niu, X.-B. Chen, and H.M. Xiong, "A new generation of energy storage electrode materials constructed from carbon dots," Materials Chemistry Frontiers, vol. 4, no. 3, pp. 729-749, 2020.

[25] D. Qu and Z. Sun, "The formation mechanism and fluorophores of carbon dots synthesizedviaa bottom-up route," Materials Chemistry Frontiers, vol. 4, no. 2, pp. 400-420, 2020.

[26] H. P. S. Castro, V. S. Souza, J. D. Scholten et al., "Frontispiece: synthesis and characterisation of fluorescent carbon nanodots produced in ionic liquids by laser ablation," Chemistry - A European Journal, vol. 22, no. 1, 2016.

[27] E. F. C. Simões, J. M. M. Leitão, and J. C. G. E. da Silva, "Carbon dots prepared from citric acid and urea as fluorescent probes for hypochlorite and peroxynitrite," Microchimica Acta, vol. 183, no. 5, pp. 1769-1777, 2016.

[28] N. M. Zholobak, A. L. Popov, A. B. Shcherbakov et al., "Facile fabrication of luminescent organic dots by thermolysis of citric acid in urea melt, and their use for cell staining and polyelectrolyte microcapsule labelling," Beilstein Journal of Nanotechnology, vol. 7, pp. 1905-1917, 2016.

[29] A. Raghavan, S. Sarkar, L. R. Nagappagari, S. Bojja, S. MuthukondaVenkatakrishnan, and S. Ghosh, "Decoration of graphene quantum dots on TiO2Nanostructures: photosensitizer and cocatalyst role for enhanced hydrogen generation," Industrial \& Engineering Chemistry Research, vol. 59, no. 29, pp. 13060-13068, 2020.

[30] T. T. Quang, N. X. Truong, T. H. Minh, N. N. Tue, and G. T. P. Ly, "Enhanced photocatalytic degradation of MB under visible light using the modified MIL-53(Fe)," Topics in Catalysis, vol. 63, no. 11-14, pp. 1227-1239, 2020.

[31] Y. Zhang, G. Li, H. Lu, Q. Lv, and Z. Sun, "Synthesis, characterization and photocatalytic properties of MIL-53(Fe)-graphene hybrid materials," RSC Advances, vol. 4, no. 15, pp. 7594-7600, 2014.

[32] C. Zhang, L. Ai, and J. Jiang, "Solvothermal synthesis of MIL$53(\mathrm{Fe})$ hybrid magnetic composites for photoelectrochemical water oxidation and organic pollutant photodegradation under visible light," Journal of Materials Chemistry A, vol. 3, no. 6, pp. 3074-3081, 2015.

[33] J. Tan, Y. Ye, X. Ren, W. Zhao, and D. Yue, "High pH-induced efficient room-temperature phosphorescence from carbon dots in hydrogen-bonded matrices," Journal of Materials Chemistry C, vol. 6, no. 29, pp. 7890-7895, 2018.

[34] C. Liu, F. Zhang, J. Hu, W. Gao, and M. Zhang, "A Mini review on $\mathrm{pH}$-sensitive photoluminescence in carbon nanodots," Frontiers in Chemistry, vol. 8, p. 1242, 2021.

[35] J. Peng, Y. Li, X. Sun et al., "Controlled manipulation of metalorganic framework layers to nanometer precision inside large mesochannels of ordered mesoporous silica for enhanced removal of bisphenol a from water," ACS Applied Materials \& Interfaces, vol. 11, no. 4, pp. 4328-4337, 2019.

[36] H.-C. Li, W.-J. Liu, H.-X. Han, and H.-Q. Yu, "Hydrophilic swellable metal-organic framework encapsulated Pd nanoparticles as an efficient catalyst for $\mathrm{Cr}(\mathrm{vi})$ reduction," Journal of Materials Chemistry A, vol. 4, no. 30, pp. 11680-11687, 2016.

[37] R. Panda, S. Rahut, and J. K. Basu, "Preparation of a Fe2O3/MIL-53(Fe) composite by partial thermal decomposition of MIL-53(Fe) nanorods and their photocatalytic activity," RSC Advances, vol. 6, no. 84, pp. 80981-80985, 2016. 
[38] C.-F. Zhang, L.-G. Qiu, F. Ke et al., "A novel magnetic recyclable photocatalyst based on a core-shell metal-organic framework Fe3O4@MIL-100(Fe) for the decolorization of methylene blue dye," Journal of Materials Chemistry A, vol. 1, no. 45, p. 14329, 2013.

[39] D. T. Sun, L. Peng, W. S. Reeder et al., "Rapid, selective heavy metal removal from water by a metal-organic framework/polydopamine composite," ACS Central Science, vol. 4, no. 3, pp. 349-356, 2018.

[40] W. Liang, L. Li, J. Hou et al., "Linking defects, hierarchical porosity generation and desalination performance in metalorganic frameworks," Chemical Science, vol. 9, no. 14, pp. 3508-3516, 2018.

[41] W. Huang, N. Liu, X. Zhang, M. Wu, and L. Tang, "Metal organic framework g- $\mathrm{C}_{3} \mathrm{~N}_{4} / \mathrm{MIL}-53(\mathrm{Fe})$ heterojunctions with enhanced photocatalytic activity for $\mathrm{Cr}(\mathrm{VI})$ reduction under visible light," Applied Surface Science, vol. 425, pp. 107-116, 2017. 